\title{
Analysis of the ColE1 stability determinant Rcd
}

\author{
Michaela E. Sharpe, † Heather M. Chatwin, Cindy Macpherson, \\ Helen L. Withersł and David K. Summers \\ Author for correspondence: David K. Summers. Tel: +44 1223 333991. Fax: +441223333992. \\ e-mail: d.summers $($ gen.cam.ac.uk
}

Department of Genetics, Downing Street, Cambridge CB2 3EH, UK

\begin{abstract}
Multimer formation is an important cause of instability for many multicopy plasmids. Plasmid ColE1 is maintained stably because multimers are converted to monomers by Xer-mediated site-specific recombination at the cer site. However, multimer resolution is not the whole story; inactivation of a promoter $\left(P_{\text {cer }}\right)$ within cer causes plasmid instability even though recombination is unaffected. The promoter directs the synthesis of a short transcript (Rcd) which is proposed to delay the division of multimer-containing cells. Mapping of the $5^{\prime}$ terminus of Rcd confirms that transcription initiates from $P_{\text {cer }}$. The $3^{\prime}$ terminus shows considerable heterogeneity, consistent with a primary transcript of 95 nt being degraded via intermediates of 79 and $70 \mathrm{nt}$. Secondary structure predictions for Rcd are presented. Of four mutations which abolish Rcd-mediated growth inhibition, one reduces the activity of $\mathbf{P}_{\text {cer }}$ while the other three map to the rcd coding sequence and reduce the steadystate level of the transcript. RNA folding analysis suggests that these three mutant transcripts adopt a common secondary structure in which the major stem-loop differs from that of wild-type Rcd. A survey of 24 cer-like multimer resolution sites revealed six which contain $P_{\text {cer }}$-like sequences. The putative transcripts from these sites have similar predicted secondary structures to Rcd and contain a highly conserved 15 base sequence. To test the hypothesis that Rcd acts as an anti-sense RNA, interacting with its target gene(s) through the 15 nt sequence, we used DNA hybridization and sequence analysis to find matches to this sequence in the Escherichia coli chromosome. Our failure to find plausible anti-sense targets has led to the suggestion that Rcd may interact directly with a protein target.
\end{abstract}

Keywords: ColE1, dimer resolution, cell cycle, anti-sense RNA, plasmid stability

\section{INTRODUCTION}

Multimer formation and associated copy number depression are acknowledged causes of cloning vector instability (Summers et al., 1993; Summers, 1998). Multimers of the naturally occurring plasmid ColE1 are resolved to monomers by site-specific recombination between directly repeated cer sites (Summers \& Sherratt, 1984). In addition to the recombination site, ColE1 multimer resolution requires four chromosome-encoded proteins: ArgR (Stirling et al., 1988), PepA (Stirling et al., 1989), XerC (Colloms et al., 1990) and XerD (Blakely et al., 1993). However, multimer resolution alone is

†Present address: Chemical Pathology Unit, Sir William Dunn School of Pathology, University of Oxford, South Parks Road, Oxford OX1 3RE, UK.

¥Present address: Department of Cell and Molecular Biology, Box 596, Biomedical Centre, Uppsala University, S-751 24 Uppsala, Sweden. insufficient to ensure stable maintenance of ColE1-like plasmids. A promoter $\left(\mathrm{P}_{c e r}\right)$ which is located centrally within the cer site (Summers \& Sherratt, 1988) is also required and mutational inactivation of this promoter results in plasmid instability, even though recombination at cer is unaffected (Patient \& Summers, 1993).

$\mathrm{P}_{c e r}$ directs the synthesis of Rcd (regulator of cell division); a short, untranslated transcript. The growth of cells overexpressing Rcd from an up-promoter derivative of $\mathrm{P}_{c e r}$ or from $\lambda \mathrm{P}_{\mathrm{R}}$ is severely retarded on solid media and microscopic examination suggests that there is a 'bottle-neck' in their cell cycle between nucleoid segregation and septation (Patient \& Summers, 1993). To explain the role of Rcd in plasmid stability it has been proposed that its expression from $P_{c e r}$ in response to plasmid multimerization delays cell division until multimer resolution is complete (Patient \& 
Summers, 1993). The need to delay cell division implies that Xer-cer recombination is relatively slow and an increasing amount of circumstantial evidence supports this view (Summers, 1998).

The mechanism of action of Rcd is unknown but some clues have come recently from studies of Rcd overexpression in broth culture. Rcd overexpression slows the growth of wild-type cells in broth but the culture eventually reaches stationary phase at a normal cell density. In contrast, Rcd induction in hns mutant cells blocks cell division and growth ceases completely after 2-3 h (Summers \& Rowe, 1997; Rowe \& Summers, 1999). The nucleoid in these so-called quiescent cells is highly condensed, but they remain capable of transcription and translation and are capable of expressing high levels of plasmid-encoded protein. The continuing metabolic activity of quiescent cells in the absence of growth suggests that Rcd inhibits cell division directly, rather than via a block on RNA or protein synthesis. We have pursued the idea that it might act as an anti-sense RNA, achieving its effect on growth by inhibiting mRNA translation. Two examples of trans-acting antisense RNAs in Escherichia coli are the $53 \mathrm{nt}$ DicF (Tétart \& Bouché, 1992), which regulates fts $Z$ mRNA, and the 93 nt MicF (Andersen \& Delihas, 1990), which inhibits translation of $o m p F$ mRNA. In both cases part of the anti-sense transcript displays complementarity to the region of the target mRNA containing the ribosomebinding site. In DicF, a region of 31 nt shows $81 \%$ complementarity to $f t s Z$ mRNA immediately upstream of the start codon. In MicF a region of 54 nt shows $80 \%$ complementarity to the corresponding region of $o m p F$ mRNA.

Searching for the target of Rcd has been difficult in the absence of precise knowledge of the $5^{\prime}$ and $3^{\prime}$ termini, or any idea of which part of the transcript is involved in the target interaction. In this report we describe the mapping of the $5^{\prime}$ and $3^{\prime}$ ends of Rcd which has facilitated prediction of its secondary structure. We have employed a bioinformatic approach to identify the active site of Rcd and have looked for complementarity to the presumed active site by hybridization and by searching the E. coli genome sequence. Our failure to find a target by either of these approaches has led us to speculate that Rcd might interact directly with a protein target.

\section{METHODS}

Bacteria and plasmids. All the bacteria used were derivatives of E. coli strain AB1157 (Bachmann, 1972). DS941 is AB1157 recF lacl" lacZ $\triangle \mathrm{M} 15$. JC8679 (AB1157 $\mathrm{rec} B C$ sbcA) was obtained from Professor D. J. Sherratt, Department of Biochemistry, University of Oxford. Plasmid pKS490 is a pUC8 derivative which has a cer site inserted into the multiple cloning site (Summers \& Sherratt, 1988). pKS494 and pKS496 are derivatives of pKS490. In pKS494 the $\mathrm{P}_{c e r}$ promoter has been inactivated by mutation of the invariant T and in pKS496 a mutation within $\mathrm{P}_{r e r}$ has increased the level of Rcd expression (Patient \& Summers, 1993). Bacteriophages M13mp8cer and M13mp19cer are derivatives of M13mp8 and M13mp19 in which the cer site from pKS490 has been inserted as an
EcoRI-HindIII fragment into the polylinker of the phage. Plasmids pHW115 and $\mathrm{pHW} 118$ are derivatives of the transcription fusion vector pKO1 (McKenny et al., 1981); their construction is described in Results.

Media and bacterial transformation. For routine growth of bacteria we used L-broth (Kennedy, 1971) and Oxoid isosensitest agar. Where appropriate, ampicillin $\left(50 \mu \mathrm{g} \mathrm{ml}^{-1}\right)$ was added to the medium. Plasmid transformation of $\mathrm{CaCl}_{2}$ treated cells was by the method of Cohen \& Hsu (1972). When transformants were to be grown at $42^{\circ} \mathrm{C}$, all stages of the procedure normally performed at $37^{\circ} \mathrm{C}$ were performed at $42{ }^{\circ} \mathrm{C}$.

Hydroxylamine mutagenesis. Plasmid DNA (40 $\mu$ l in sterile distilled water) was mixed with $20 \mu \mathrm{l} 0 \cdot 1 \mathrm{M}$ potassium phosphate buffer $(\mathrm{pH} \mathrm{6.0)}$ containing $5 \mathrm{mM}$ EDTA and prewarmed to $75^{\circ} \mathrm{C}$. Forty microlitres of a freshly prepared solution of $1 \mathrm{M}$ hydroxylamine $(0.56 \mathrm{ml} 4 \mathrm{M} \mathrm{NaOH}, 0.35 \mathrm{~g}$ hydroxylamine hydrochloride, made up to $5 \mathrm{ml}$ in water) was pre-heated for $5 \mathrm{~min}$ at $75^{\circ} \mathrm{C}$ and the two solutions were mixed and incubated at $75^{\circ} \mathrm{C}$ for $60 \mathrm{~min}$. Sterile distilled water $(400 \mu \mathrm{l})$ and cold 2-propanol $(500 \mu \mathrm{l})$ were added and the mixture was placed on ice for $10 \mathrm{~min}$. After centrifugation (13000 r.p.m. for $10 \mathrm{~min}$ ) the supernatant was discarded and the DNA pellet washed twice with $70 \%$ ethanol. After drying the pellet was dissolved in $20 \mu \mathrm{T}$ TE.

Isolation of RNA and Northern blotting. Methods described in Patient \& Summers (1993) were used to isolate RNA, to prepare the oligonucleotide probe and to perform Northern blotting.

$3^{\prime}$ and $5^{\prime}$ end-mapping of RNA. $3^{\prime}$ end-mapping of RNA was performed according to the method of Maniatis et al. (1982). The DNA probe was made by primer extension on M13mp8cer from the -40 forward primer. This produced a radiolabelled strand complementary to $\mathrm{Rcd}$. The doublestranded primer extension products were cleaved with Pvul (CGATCG) which cuts 9 bp downstream of the Rcd transcription start (see Fig. 2). The probe was eluted from a polyacrylamide gel by the 'crush and soak' method (Maniatis et al., 1982). For digestion of the probe-RNA hybrid Maniatis et al. (1982) recommend $100-1000$ units $S 1$ nuclease $\mathrm{ml}^{-1}$ but to ensure complete digestion of single-stranded regions we explored a range of 1000-3500 units S1 nuclease $\mathrm{ml}^{-1}$ at an incubation temperature of $40^{\circ} \mathrm{C}$. Our results indicated that under our conditions digestion was complete at above 2000 units $\mathrm{S} 1$ nuclease $\mathrm{ml}^{-1}$. Digestion products were subjected to electrophoresis on an $8 \%$ polyacrylamide $/ 7 \mathrm{M}$ urea gel beside a standard dideoxy sequencing reaction using M13mp19cer as template and a sequencing primer whose $5^{\prime}$ end was exactly complementary to the $3^{\prime}$ end of our S1 mapping probe $\left(5^{\prime}\right.$ ATCGCGGCAGTTTTTCG-3').

To map the $5^{\prime}$ end of Rcd, reverse transcriptase was used to synthesize DNA from a primer complementary to an internal region of the transcript. The DNA strand terminates at the $5^{\prime}$ end of the transcript. Labelled oligonucleotide primer (5$10 \mathrm{ng}$ ) was annealed to $5 \mathrm{ng}$ total cellular RNA in the presence of $1 \mathrm{M} \mathrm{KCl}(1 \mu \mathrm{l})$. The mixture was heated to $90^{\circ} \mathrm{C}$ for $5 \mathrm{~min}$ and cooled slowly to $42^{\circ} \mathrm{C}$ over a $2 \mathrm{~h}$ period (all subsequent procedures were performed at $42{ }^{\circ} \mathrm{C}$ to minimize the problems of secondary structure). Four microlitres of $5 \times$ reverse transcriptase buffer $(250 \mathrm{mM}$ Tris $/ \mathrm{HCl}, \mathrm{pH} 8.3,375 \mathrm{mM} \mathrm{KCl}$, $50 \mathrm{mM}$ DTT, $15 \mathrm{mM} \mathrm{MgCl}_{2}$ ) and $1 \mu \mathrm{l}$ distilled water were added to the annealed mixture and incubated at $42^{\circ} \mathrm{C}$ for $30 \mathrm{~min}$. Primer extension was performed by adding $4 \mu \mathrm{l} \mathrm{dNTP}$ mixture (2.5 mM each dNTP adjusted to $\mathrm{pH} \mathrm{7.5)}$ and 200 units $M-M L V$ reverse transcriptase and continuing the incubation 
for $2 \mathrm{~h}$ at $42^{\circ} \mathrm{C}$. After $1 \mathrm{~h}$ a further 200 units reverse transcriptase was added. Twenty microlitres of STOP mix (95\% formamide, $20 \mathrm{mM}$ EDTA, 0.05\% bromophenol blue, $0.05 \%$ xylene cyanol) was added to terminate the reaction. An ATCG ladder was obtained with the same primer oligonucleotide (unlabelled) using plasmid DNA as the template for sequencing. The products of both the primer extension analysis and sequencing reactions were denatured prior to loading on a sequencing gel. Autoradiography was at $-70^{\circ} \mathrm{C}$.

Probing the E. coli Gene Mapping Membrane. In a direct approach to identify the target of Rcd, an oligonucleotide complementary to the 15 base conserved region of Rcd was end-labelled with $\left[\gamma^{-3} \mathrm{P}\right] \mathrm{ATP}$ and hybridized to the Gene Mapping Membrane (Noda et al., 1991). The hybridization was carried out under conditions of very low stringency (i.e. at $40{ }^{\circ} \mathrm{C}$ with the melting temperature of probe estimated to be $\left.48^{\circ} \mathrm{C}\right)$ in a buffer consisting of $6 \times$ SSC $(0.9 \mathrm{M}$ sodium chloride, $0.09 \mathrm{M}$ sodium citrate) and $0.5 \%$ SDS without blocking agents. Following hybridization, the membrane was given a $5 \mathrm{~min}$ rinse in $6 \times \mathrm{SSC}, 0.1 \%$ SDS before exposing to $\mathrm{X}$-ray film at $-70{ }^{\circ} \mathrm{C}$.

\section{RESULTS}

\section{5 ' end-mapping of Rcd}

Rcd appears to be produced from the $\mathrm{P}_{c e r}$ promoter since mutation of the invariant $T$ in the -10 sequence abolishes Rcd expression (Patient \& Summers, 1993). To establish the transcription start site we used primer extension by reverse transcriptase to map the $5^{\prime}$ ends of Rcd expressed from plasmids pKS490 and pKS496. The cer site in pKS490 contains the wild-type $\mathrm{P}_{c e r}$ promoter and $r c d$ sequence; pKS496 is identical except for an uppromoter mutation in $P_{c e r}$ (Fig. 1a; Patient \& Summers, 1993). Since $P_{c e r}$ is activated by plasmid multimerization, total RNA was prepared from pKS490 or pKS496 in JC8679; a recBC sbcA strain in which both plasmids are extensively multimeric. The primer for reverse transcriptase was an oligonucleotide complementary to a sequence approximately $30 \mathrm{nt}$ downstream of $\mathrm{P}_{c e r}$ (Fig. 2). Primer extensions on RNA from strains carrying either plasmid yielded major products of 25, 26 and $27 \mathrm{nt}$. The result for JC8679(pKS496) is shown in Fig. 1(b). The intensities of the bands suggest that three start points are used at similar frequencies. The corresponding $5^{\prime}$ ends of Rcd were identified by comparing the primer extension products with the products of a DNA sequencing reaction using the same primer and are marked with asterisks in Fig. 1(a).

\section{3' end-mapping of Rcd}

In an earlier study (Patient \& Summers, 1993) it was proposed that $r c d$ transcription terminates at a sequence resembling a $\rho$-independent terminator $70-80$ bases downstream of the transcription start. To establish the termination site more directly, S1 mapping of the Rcd 3' end was performed on total RNA from JC8679(pKS490) (wild-type $\mathrm{P}_{\text {cer }}$ ). We used a radiolabelled ssDNA probe complementary to Rcd, whose $3^{\prime}$ end was 9 nt downstream of the transcription start. We chose a probe which does not cover the $5^{\prime}$ end of Rcd so that variability (a)

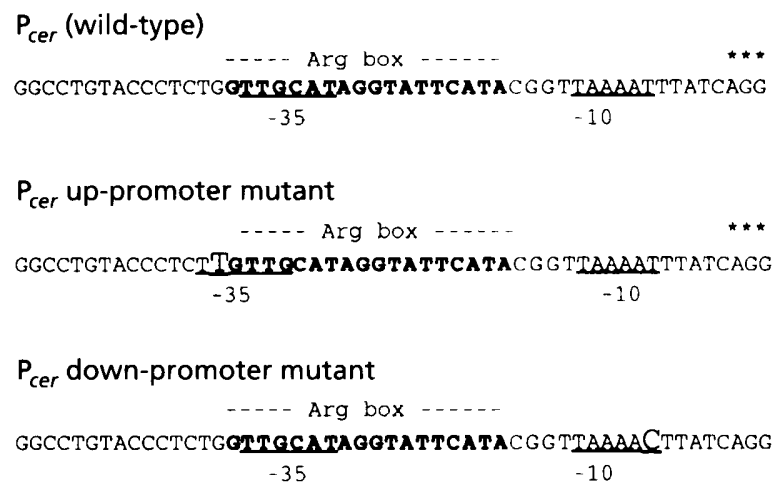

(b)

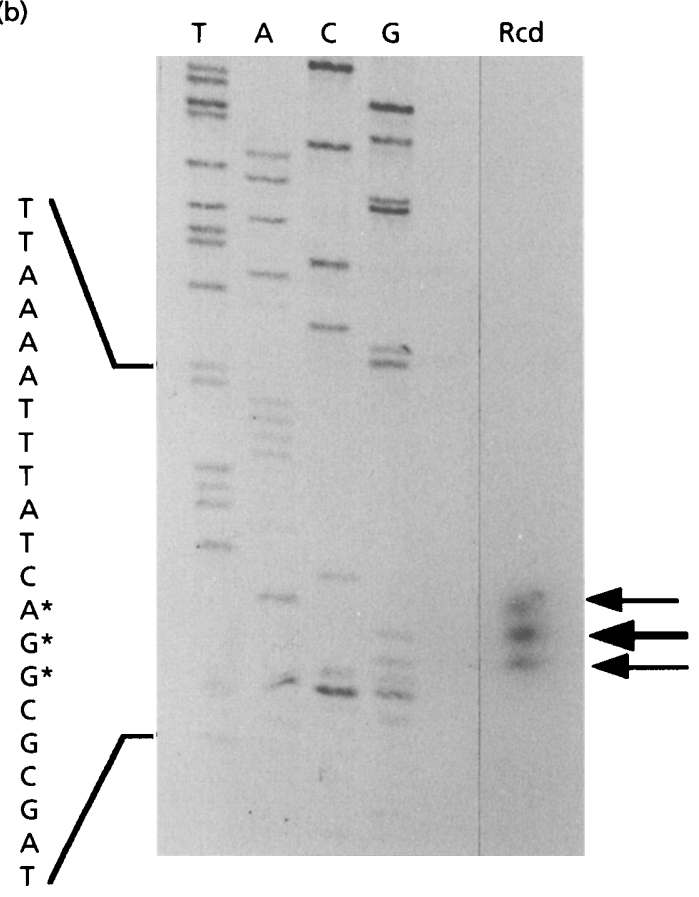

Fig. 1. (a) Sequences of the $P_{c e r}$ promoter and its derivatives. The -10 and -35 sequences are underlined and the binding sites of ArgR (Arg boxes) are in bold type. Transcription start sites are indicated by asterisks and the altered bases in the upand down-promoter derivatives are enlarged. (b) $5^{\prime}$ endmapping of Rcd transcribed from the $P_{\text {cer }}$ up-promoter derivative in pKS496. Equivalent results were obtained with the wild-type promoter (data not shown).

of the transcription start site did not influence the result of the $3^{\prime}$ end-mapping. S1 nuclease digestion of the Rcd-probe hybrid should yield a labelled DNA fragment whose $5^{\prime}$ end is complementary to the $3^{\prime}$ end of the Rcd transcript. The products of digestion were run on a sequencing gel alongside a cer sequence primed from an oligonucleotide whose $5^{\prime}$ end was exactly complementary to the $3^{\prime}$ end of the DNA probe. The $3^{\prime}$ ends of Rcd could thus be inferred directly from the products of the sequencing reaction. The result of the analysis was complex but highly reproducible over eight independent 

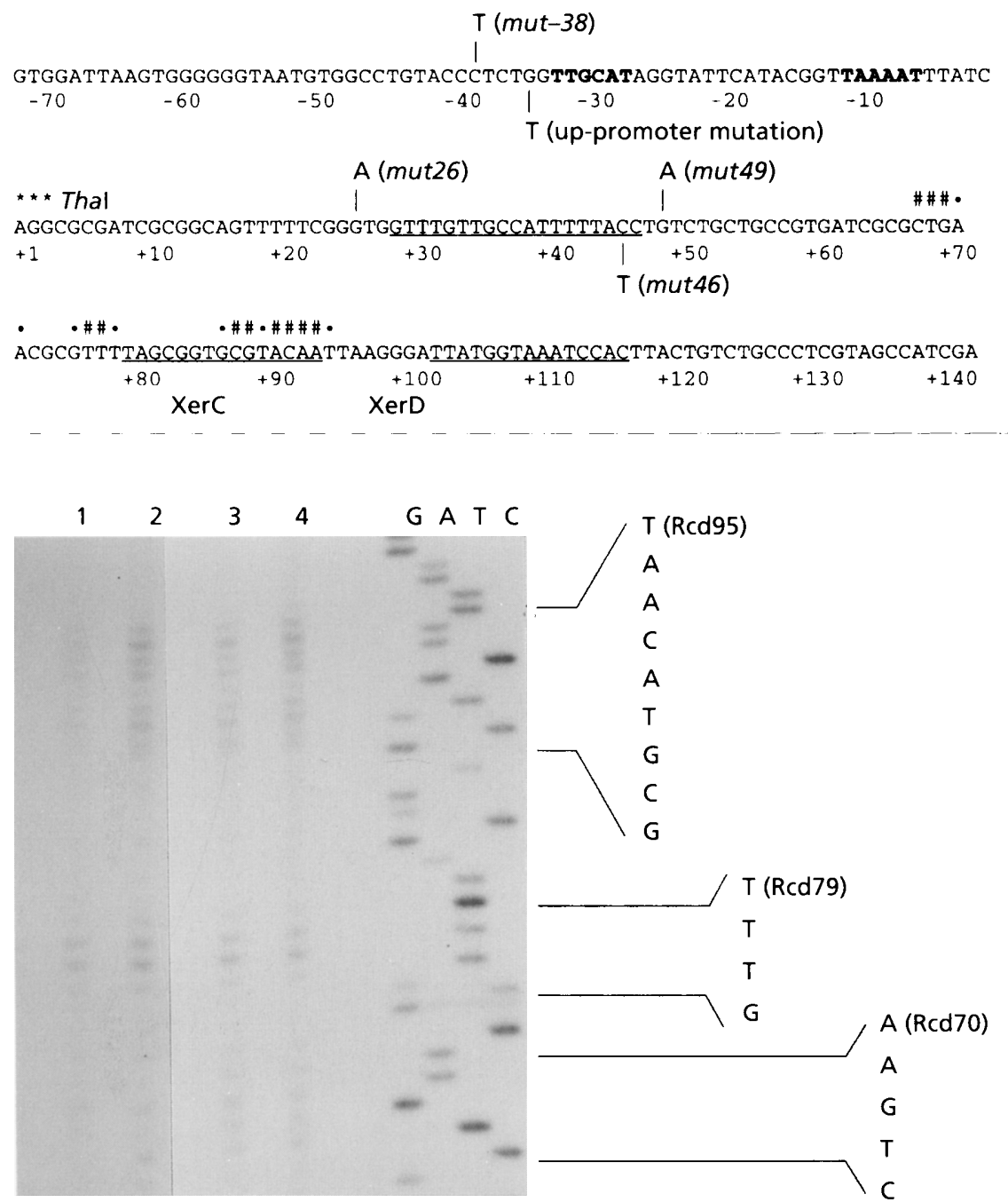

Fig. 2. Part of ColE1 cer showing $P_{\text {cer }}(-10$ and -35 sequences in bold type), the Rcd coding region and the XerC and XerD binding sites. Asterisks indicate transcription start sites $(* * *)$. The results of $3^{\prime}$ endmapping of Rcd (see Fig. 3) are summarized as follows: \#, a strong signal; $\bullet$, weak signal. Mutations which abolish Rcdmediated growth inhibition are shown above or below the main sequence, followed by the mutant number in parentheses. The Thal site immediately downstream of $P_{c e r}$ used for cloning promoter fragments into pKO1 is shown and the sequence complementary to the oligonucleotide used for 5 ' end-mapping of Rcd is underlined. experiments; typical results are shown in Fig. 3. We observed one major cluster of bands $(\operatorname{Rcd} 95)$ and two minor clusters $(\operatorname{Rcd} 79$ and $\operatorname{Rcd} 70)$. At least half of the 3' termini appear to be within the Rcd95 cluster. The distribution of Rcd 3' termini is summarized in Fig. 2.

\section{Predicting the secondary structure of Rcd}

The transcript mapping results show heterogeneity at both the $3^{\prime}$ and $5^{\prime}$ ends and suggest that cells expressing Rcd contain a family of transcripts between 68 and $95 \mathrm{nt}$. This is consistent with the previous observation that Northern analysis of Red on low resolution gels gives a smear of approx. 70-90 nt (Patient \& Summers, 1993; but note that in Fig. 3 of this publication the size markers were misaligned). We used MFOLD from the GCG package to predict the secondary structures of three representative Rcd transcripts (Fig. 4). The input sequences started at the first base of the AGG initiation triplet and terminated at bases $95(\operatorname{Rcd} 95), 79(\operatorname{Rcd} 79)$ and $70(\operatorname{Rcd} 70)$, which correspond to the slowest- running band in each of the three clusters of $3^{\prime}$ ends identified by $\mathrm{S} 1$ mapping (Fig. 3). The predicted structures for $\operatorname{Rcd} 70$ and $\operatorname{Rcd} 79$ contain a single stem-loop while an additional minor stem-loop appears in Rcd95. The large GC-rich stem-loop is identical in all three structures and is interrupted by an internal loop and an asymmetrical bulge. MFOLD offered alternative structures of very similar energy but with a loop of $13 \mathrm{nt}$ (see, for example, Fig. 4 of Patient \& Summers, 1993). Such a large loop seemed less plausible than the $5 \mathrm{nt}$ loop structure which is close to the optimum for regulator-target interactions (Hjalt \& Wagner, 1992). However, final confirmation of Rcd secondary structure must await physical mapping of the transcript.

\section{Characterization of mutations which reduce Red effectiveness}

In an attempt to learn more about the relationship between Rcd structure and function, we screened for mutations in the transcript which reduce its effectiveness 


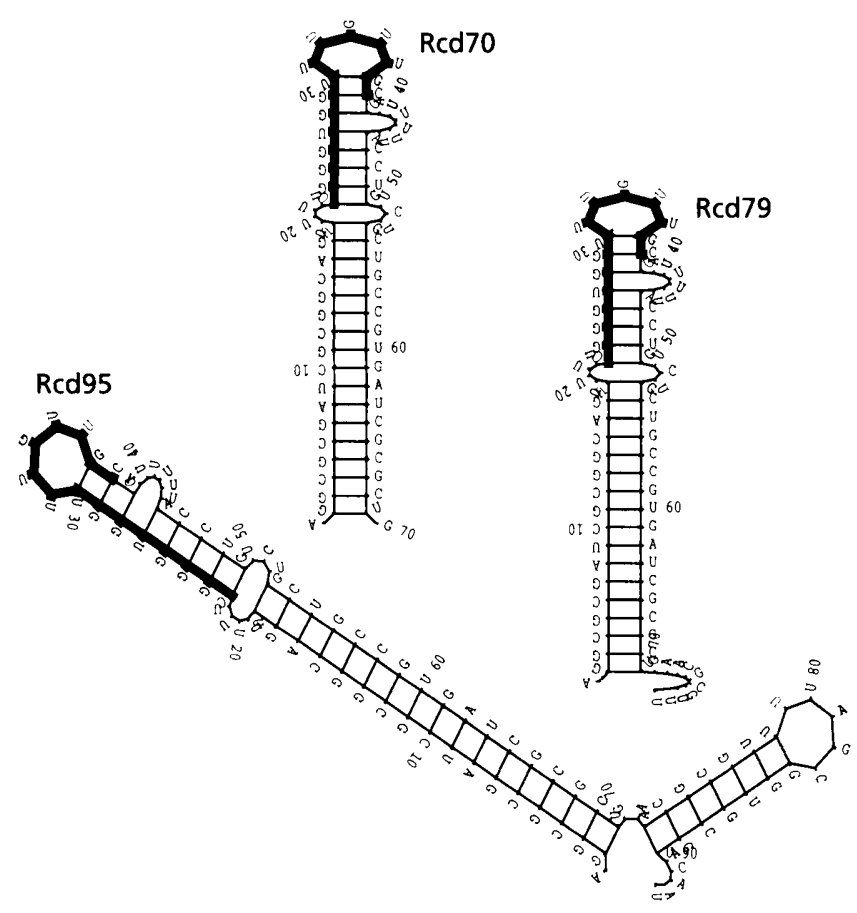

Fig. 4. Computer-predicted secondary structures for Rcd. The structures have a common $5^{\prime}$ end and $3^{\prime}$ termini at nt 95 (Rcd95), 79 (Rcd79) or 70 (Rcd70). The 15 nt sequence conserved among six hypothetical Rcd-like transcripts is indicated by a thick line.

as a growth inhibitor. Plasmid pKS496 overexpresses Rcd because of an up-promoter mutation in $\mathrm{P}_{c e r}$ and consequently DS941(pKS496) can only form minute colonies on L-agar after overnight incubation at $42{ }^{\circ} \mathrm{C}$ (Patient \& Summers, 1993). Hydroxylamine-mutagenized pKS496 was transformed into DS941 and the selection plates were incubated overnight at $42{ }^{\circ} \mathrm{C}$ when a small number of normal-sized colonies appeared. Plasmid DNA from these colonies was transformed into DS941 and the normal growth of transformed colonies at $42{ }^{\circ} \mathrm{C}$ confirmed that a plasmid mutation was responsible for the loss of Rcd-mediated growth inhibition. Four mutant plasmids were sequenced and a single base change (in addition to the $\mathrm{P}_{c e r}$ up-promoter mutation) was identified in each (Fig. 2). They were all within cer; three (mut26, mut46 and mut49) within rcd and one (mut-38) upstream of $\mathrm{P}_{c e r}$. Each mutation is identified by a number which refers to its position relative to the first base of the AGG trinucleotide where transcription initiates. The cer sites in all of the mutant plasmids remained proficient in dimer resolution and measurement of plasmid copy number confirmed that the loss of growth inhibition was not due to a reduction in the number of Rcd-producing plasmids (data not shown).

All three mutations in the $r c d$ coding region alter bases in the upper part of the major stem-loop of the predicted structure. mut 26 and mut 46 change bases which are paired in the wild-type structure. Hypothetical (a)

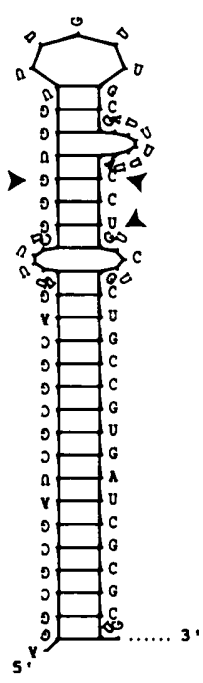

Rcd wild-type

(b)

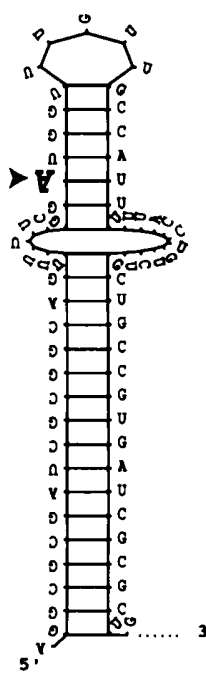

mut26

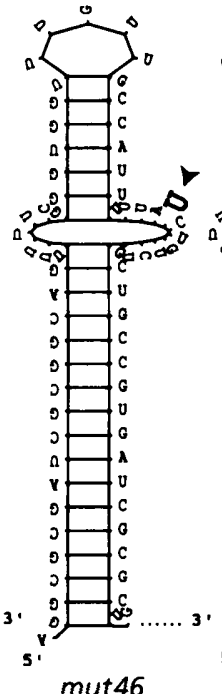

mut46

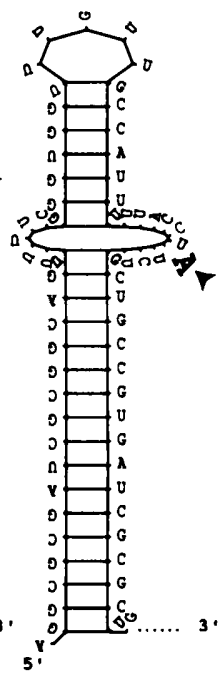

mut49
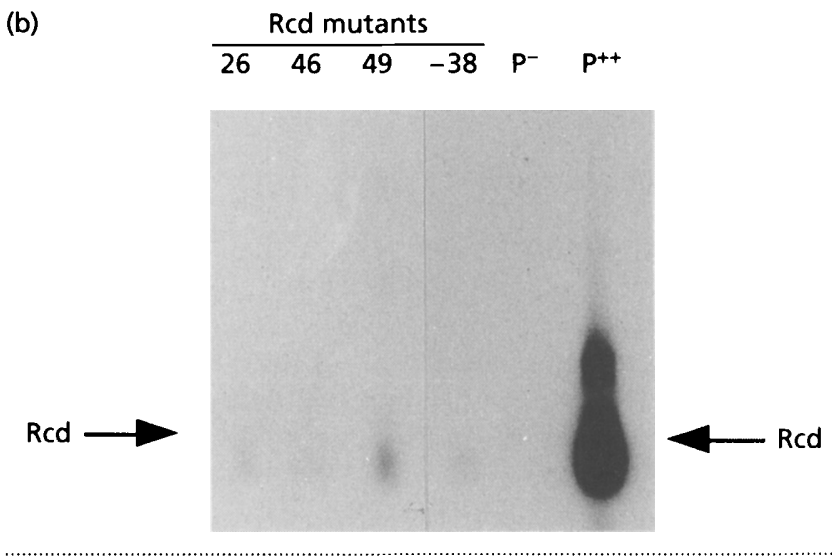

Fig. 5. (a) Predicted structures of wild-type Rcd and mutant transcripts mut26, mut46 and mut49. The altered bases in the mutant transcripts are enlarged and marked with arrowheads. The corresponding positions in the wild-type structure are marked with arrow heads. (b) Northern blots of total RNA from cultures of JC8679 expressing mutant derivatives of Rcd. Lane numbers refer to the mutation co-ordinate (see Fig. 2). $\mathrm{P}^{-}, \quad J$ C8679(pKS494) (down-promoter mutation in $\mathrm{P}_{\text {cer) }}$ ); $\mathrm{P}^{*}$ ", JC8679(pKS496) (up-promoter mutation in $\mathrm{P}_{\text {cer }}$ ).

structures for the three mutant Rcd transcripts were generated using MFOLD (Fig. 5a). Despite the differences in their primary sequences, the predicted structures for the mutant transcripts are identical. Compared to the wild-type structure prediction, the upper part of the major stem is shorter and the bulge and internal loop are replaced by a single large internal loop. The loss of Rcdmediated growth inhibition in these three mutants could result from instability of the transcript or a reduction in the effectiveness of its target interaction. Instability would result in a reduction of the steady-state level of transcript, so we compared levels of Rcd in total RNA from JC8679(pKS496) and each of the mutant plasmids. Equal amounts of total RNA were run on a low resolution gel and a Northern blot was probed with the 
Table 1. Galactokinase activity expressed from $P_{c e r}$ fused to galK

\begin{tabular}{|lc|}
\hline Strain & $\begin{array}{c}\text { Galactokinase } \\
\text { activity* } \\
\text { (mean } \pm \text { SD) } \\
\text { [no. of replicas] }\end{array}$ \\
\hline DS941(pKS463) & $61 \pm 24[31]$ \\
DS94largR(pKS463) & $46 \pm 14[14]$ \\
DS941(pHW115) & $242 \pm 65[11]$ \\
DS941(pHW118) & $158 \pm 63[12]$ \\
DS94largR(pHW 115) & $411 \pm 91[12]$ \\
DS94largR(pHW 118) & $208 \pm 122[8]$ \\
\hline
\end{tabular}

* Galactokinase units as defined by McKenny et al. (1981).

oligonucleotide used for $5^{\prime}$ end-mapping of Rcd. A strong Rcd band was present in the pKS496 track but was barely detectable in RNA from cultures containing the mutant plasmids (Fig. 5b).

\section{Mut-38 reduces the activity of $\mathbf{P}_{\text {cer }}$}

The fourth mutation (mut -38 ) was a $\mathrm{C}$ to $\mathrm{T}$ change upstream of $\mathrm{P}_{c^{\rho} r}$ at ColE1 co-ordinate 3790 (Fig. 2). Northern analysis of total RNA showed a dramatic reduction in the steady-state level of Rcd (Fig. 5b). It has been known for some time that sequences upstream of $P_{r e r}$ influence transcription (Summers \& Sherratt, 1988) so it seemed plausible that $m u t-38$ down-regulates Rcd expression. To test this, transcription fusion plasmids were constructed containing $\mathrm{P}_{\text {cer }}$ from pKS496 and its mut -38 derivative upstream of a promoterless galK gene in pKO1 (pHW115 and pHW118, respectively). The inserts in these fusion plasmids contain the whole of the cer site upstream of $P_{r e r}$ and stop at the Thal site immediately downstream of the transcription start (Fig. 2). In DS941, pHW118 showed a lower level of transcription than pHW $115 \quad(158 \pm 63$ and $242 \pm 65$ galactokinase units, respectively). Although the reduction in transcription detected in the gene fusion experiment is small compared with the much greater decline in Red concentration seen by Northern blotting, it seems likely that reduced transcription from the mut-38 promoter is responsible for the lifting of growth inhibition.

ArgR is a transcriptional repressor of genes for arginine biosynthesis and an essential accessory factor in ColE 1 dimer resolution. The ArgR binding site (the Arg box) overlaps the -35 consensus of $P_{r e r}$ (Fig. 1a) and, noticing the proximity of the $m u t-38$ mutation to the Arg box, we wondered whether it might exert its effect by altering the binding of $\operatorname{ArgR}$ to $\mathrm{P}_{c o r}$. Experiments with pKS463 (Summers \& Sherratt, 1988) in which wildtype $P_{r e r}$ is fused to galK (Table 1) showed that the presence or absence of ArgR had little effect. To investigate the effect of $\mathrm{ArgR}$ on the mutant promoters, transcription from $\mathrm{P}_{\text {cer }}$ in $\mathrm{pHW} 118$ and $\mathrm{pHW} 115$ was compared in DS941 and DS941argR. The cer sites in both of these plasmids contain the $\mathrm{P}_{c e r}$ up-promoter mutation and in addition pHW 118 contains mut -38 . The absence of $\operatorname{ArgR}$ increased promoter activity for both plasmids (Table 1) but transcription from pHW118 was still lower than from pHW115. Thus it seems unlikely that the effect of $m u t-38$ is due simply to altered binding of ArgR to the promoter.

\section{rcd-like genes in other multimer resolution sites}

In an attempt to identify the active site of Rcd (i.e. that part of the molecule which interacts with the target) we have investigated Rcd analogues among 24 cer-like multimer resolution sites available on DNA sequence databases. Although functional Rcd analogues might diverge at the primary sequence level, we would expect to see conservation of secondary structure. Furthermore, there should be conservation of primary sequence within the active site. As a first step we searched the 24 multimer resolution sites for $\mathrm{P}_{c e r}$-like promoters. In the region corresponding to the -10 sequence of $\mathrm{P}_{c e r}$ only six of the sites (those from plasmids ColE1, pWQ799, pRI13, ColK, ColA and CloDF13) retained the invariant T (Fig. 6). Mutation of this base abolishes transcription from $P_{c e r}$ (Patient \& Summers, 1993) so the remaining 18 sites seem unlikely to produce a transcript (ColN and pNTP16 are included in Fig. 6 as representatives of these sites). The six T-containing sites show complete homology to $P_{c e r}$ within their -10 sequences (TAAAAT) and strong conservation in the -35 sequence (NTGcAT). There is more sequence variation among the spacer regions but all are $15 \mathrm{nt}$ long; a characteristic feature of the $\mathrm{P}_{\text {cer }}$ promoter. Assuming the same $5^{\prime}$ and $3^{\prime}$ ends as Rcd, the putative transcribed regions in the six sites show considerable sequence variation but it is notable that one $15 \mathrm{nt}$ block (5'-CGGGTGGTTTGTTGC-3') is totally conserved, except in CloDF13 where it differs at one position. There is far less conservation of this sequence among the remaining 18 sites (exemplified by ColN and pNTP16 in Fig. 6).

In the Rcd95 secondary structure prediction the conserved $15 \mathrm{nt}$ block lies within the major stem-loop, comprising the loop and the $5^{\prime}$ side of the stem (Fig. 4). For comparison, MFOLD was used to predict secondary structures for the hypothetical transcripts from ColA, ColK and CloDF13. Despite considerable primary sequence divergence (ColE1 and CloDF 13 show only $63 \%$ identity in Rcd95) the secondary structures are similar, each displaying a major and a minor stem-loop (Fig. 7). The lower parts of the major stems vary in length from 14 to 17 bp but are invariably GC-rich. Each major stem contains two regions where base pairing is disrupted; a bulge with unpaired bases on one strand and an internal loop involving unpaired bases on both strands. In all four structures the conserved $15 \mathrm{nt}$ block begins at the internal loop on the $5^{\prime}$ side of the stem, extending through the terminal loop and ending at the bulge on the 3' side of the stem. 


\begin{tabular}{|c|c|}
\hline & -10 \\
\hline COLE1 & GGCCTGTACCCTCTGGTTGCATAGGTATTCATACGGTTAAAATTAT-C \\
\hline pWQ799 & GGTCTGTACCATCTGGTTGCATAGGTATTCATGCGGTTAAAATTTAT-C \\
\hline pRI13 & GGTCTGCACCATCTGGTTGCATAGGTATTCATACGGTTAAAATTTAT-C \\
\hline COLK & AGCGTTATTCACATGGCTGAATAGCTATGCATACGGTTAAAATTTAT-C \\
\hline COLA & GCACTGATTCACCTGGCTGCATGGTTATGCAGTCGGGTAAAATTTAT-C \\
\hline CLODF 13 & TATGCTATGCAGTTGCETGCATAGGCATGCATTAGGATAAAATTTAC-C \\
\hline COLN & CATTCAGCTTTCG? \\
\hline pNTP16 & ATCGCCATTCACTTTCATGCATAGCTATGCAGTGAGCTGAAAGCGATC \\
\hline
\end{tabular}

\begin{tabular}{|c|c|c|}
\hline & conserved region & \\
\hline COLE1 & AGGCGCGATCGCGGCAGTTTTT CGGGTGGTTTGTTGC & CATTTTTACCTGT \\
\hline pWQ799 & AGGCGCGATCGCGGCAGTTTTT CGGGTGGTTTGTTGC & CATTTTTACCTGT \\
\hline pRI13 & AGGCGCGATCGCGGCAGTTTTT CGGGTGGTTTGTTGC & CATTTTTACCTGT' \\
\hline COLK & AGGTGCGATCGCGGCA--TTTT CGGGTGGTTTGTTGC & CATTTTTACCTGT \\
\hline COLA & AGGTGCGTTCGGGGCGGTTTAC CGGGTGGTTTGTTGC & CGGTTTTACCTGT \\
\hline CLODF 13 & GGGCGCGTTTCCGGCGGTTTTC CGGGTGGGTIGTTGC & TTGTTTTATCCCG \\
\hline & $A C$ & гTTTTе \\
\hline DNTP16 & GGCATTTTTCCGGTT & ГTTTTGCGC \\
\hline
\end{tabular}

putative terminator

COLE1 CTGCTGCCGTGATCGCGCTGAACGCGTTTTA-GCGGTGCGTACAAT pWQ799 CTGCTGCCGTGATCGCGCTGAACGCGTTTTA-GCGGTGCGCCCAAG PRI13 CTGCTGCCGTGATCGCGATGAACGCGTTTTA-GCGGTGCGTACAAT COLK CAGCAGCCGTGATCGCGCTGAACGCGTTTCA-GCGGTGCGTACAAT COLA CTTCCGCCCCGAACGCAGTGAACACGTCCGGAGCGGTGCCGACAAC CLODF13 TAGCCGCCGGAAACGCCGCCAGTGCCTTACTGGCGGTACCGATAAG

COLN TTAC-GTCAGAAT-GCGTTCA--CCGTTTTG-GCGGTGCGTACAAT pNTP16 CTGC-GTCAGAATCGCGTTCAGCGCGTTTTG-GCGGTGCGCGTAAT
Fig. 6. Alignment of regions corresponding to $P_{\text {cer }}$ and red from ColE1 and multimer resolution sites in various multicopy plasmids. Asterisks $(\star \star \star)$ indicate the Rcd transcription start site.

\section{Identification of possible Rcd targets}

By virtue of its sequence conservation and position in the predicted secondary structures, the $15 \mathrm{nt}$ block has the features we would expect for the active site of Rcd. If $\mathrm{Rcd}$ regulates translation in a manner similar to $\mathrm{MicF}$ and DicF, we would expect the $15 \mathrm{nt}$ sequence to be complementary to the $5^{\prime}$ end of the target transcript in the vicinity of the ribosome-binding site. The regions of $\mathrm{MicF}$ and DicF which bind their targets contain blocks of $\mathrm{C}+\mathrm{U}$-rich sequence which bind to the $\mathrm{G}+\mathrm{A}$-rich ribosome-binding site, but no such sequence is apparent in the $15 \mathrm{nt}$ conserved block in Rcd. However this does not exclude the possibility of translational regulation since it is possible that Rcd might bind adjacent to (rather then overlapping) the ribosome site. We therefore probed an $E$. coli chromosome library and searched the $E$. coli genome sequence in an attempt to identify regions complementary to the conserved sequence. A radiolabelled DNA probe matching the $15 \mathrm{nt}$ conserved sequence was hybridized to the $E$. coli Gene Mapping Membrane (Noda et al., 1991). The membrane consists of an ordered array of overlapping phage $\lambda$ clones containing $E$. coli DNA from the Kohara library, allowing the map position of any clone to be determined rapidly. The membrane was probed on three separate occasions under conditions of very low stringency but no specific hybridization was detected.

We searched the $E$. coli genome sequence for matches to the conserved $15 \mathrm{nt}$ block but no chromosomal locus showed full homology. A contiguous 14 base match was found in the sense strand of glt $S$ at $82.4 \mathrm{~min}$, although a sense strand match is inconsistent with antisense regulation of translation. Thirteen base matches were found in the antisense strand of hns $(27.8 \mathrm{~min})$ and in an intergenic region at $35.4 \mathrm{~min}$ (Fig. 8). The match at $27.8 \mathrm{~min}$ is complementary to the extreme $5^{\prime}$ end of $\mathrm{H}$ NS mRNA (Fig. 8a) and, encouraged by this circumstantial evidence for translational control, we assayed the effect of Rcd on expression of hns-lacZ fusions. We were, however, unable to detect any effect of Rcd on the expression of either transcriptional or translational fusions, irrespective of whether the fusions were located on the chromosome or on a plasmid (data not shown).

At 35.4 min the 13 base match to the Rcd conserved region lies between two converging ORFs, an unlikely situation for the action of an anti-sense RNA. Closer inspection of the chromosome sequence revealed that the match extends beyond $r c d$ and includes the Arg box and XerC-XerD binding site of cer, suggesting that we had identified a cer-like site in the chromosome terminus region (Fig. 8b). This site is distinct from dif (Kuempel et al., 1991) which maps at $34.2 \mathrm{~min}$. Preliminary experiments in this laboratory (M. Martin, H. Griffiths \& D. K. Summers, unpublished) demonstrate that this site supports XerCD-mediated recombination when located on a plasmid. 
(a)

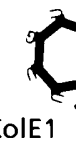

(b)

(c) ColA (76\%)

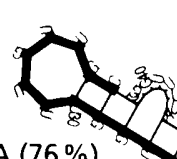

nes

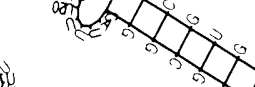

(b) ColK $(96 \%)$

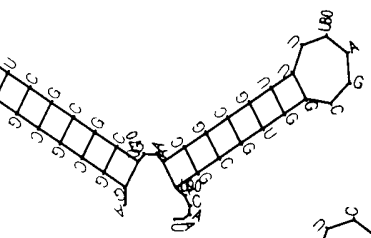

$\int_{\infty}^{\infty}$

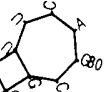

(d) CloDF13 (63\%)
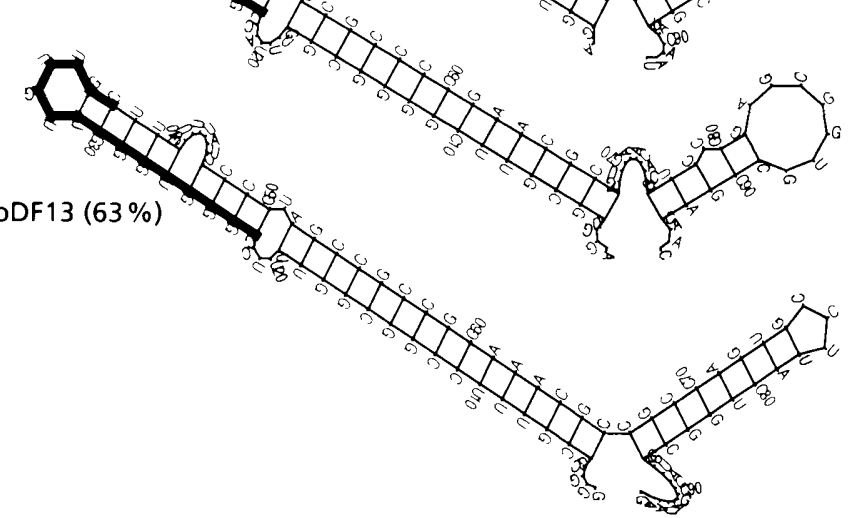

Fig. 7. Hypothetical secondary structures for ColE1 Rcd (a) and related transcripts encoded by ColK (b), ColA (c) and CloDF13 (d). The percentage sequence identity with ColE1 Rcd is shown in parentheses. A thick line marks the location of the $15 \mathrm{nt}$ conserved sequence.

\section{DISCUSSION}

\section{The structure of Rcd}

Patient \& Summers (1993) proposed a structure for Rcd based upon the assumptions that transcription starts from the $P_{c e r}$ promoter and stops at a putative $p$ independent terminator (Fig. 6) approximately 75 bases downstream. $5^{\prime}$ end-mapping of Rcd has confirmed that transcription starts at $P_{c e r}$ but $S 1$ mapping revealed a population of $\mathrm{Rcd}$ molecules with heterogeneous $3^{\prime}$ termini. The simplest interpretation of the data is that Rcd95 is the primary transcription product and the Rcd79 and Rcd70 clusters represent relatively stable intermediates during $\mathrm{Rcd}$ turnover. Heterogeneity within the three clusters may reflect exonuclease action at their $3^{\prime}$ ends or even polyadenylation of the transcripts. Termination of $\operatorname{Rcd} 95$ must occur beyond the putative terminator which, in any case, is not conserved among the six related multimer resolution sites which contain $\mathrm{P}_{c e r}$-like promoters. Interestingly, there is no terminator-like sequence corresponding to the $3^{\prime}$ end of Rcd 95 but termination occurs within the XerCD binding site (Fig. 2) and might involve the interaction of RNA polymerase with the recombinase heterodimer bound to the DNA. Alternatively, termination may occur at a downstream terminator followed by very rapid processing of the $3^{\prime}$ end.

A bioinformatic analysis identified five possible Rcd analogues in cer-like multimer resolution sites. We are optimistic about the validity of this exercise for two reasons. First, all of the sites containing a $\mathrm{P}_{c e r}$-like promoter sequence also contained a conserved 15 base sequence within the $r c d$ coding region. This sequence was not conserved among promoterless sites. Assuming that the transcripts have a common function, the $15 \mathrm{nt}$ sequence is a strong candidate for the transcript domain which makes the primary interaction with the target. Second, it is notable that secondary structure is largely conserved among the putative transcripts, despite variation in primary sequence. For example, nt 5-18 of Rcd and the corresponding region of the hypothetical CloDF13 transcript both form the lower part of the major stem. The ColE1 and CloDF13 sequences differ in four positions but in each case complementary changes in the other half of the stem ensure that base pairing is not lost. This evidence for concerted evolution of the stem sequence supports the idea that the secondary structure of Rcd and its analogues is of functional significance.

In view of the conservation of secondary structure among Rcd and its putative analogues it is interesting that the three mutations which abolish growth inhibition map to the upper stem of Rcd and computer predictions suggest that they change its structure in identical ways. Northern blot analysis of total cellular RNA from cultures bearing the mutant plasmids showed that the steady-state concentrations of these three mutant transcripts were extremely low. The steady-state concentration is determined by the rate of transcription and the half-life of the transcript but since the mutant transcripts have unaltered promoters (and therefore, presumably, unaltered rates of transcription initiation) their lower concentration must be due to more rapid turnover. Turnover rates are determined by the thermodynamic stability of the RNA, particularly the stem domain (Case et al., 1990), and susceptibility to exoribonuclease and endoribonuclease attack (Belasco et al., 1986). Since the predicted structures for the mutant transcripts are identical, this structure may be particularly susceptible to attack by cellular RNase. It is possible that wild-type Rcd may adopt this structure during turnover (e.g. as a result of interaction with an RNA-binding protein).

\section{What is the target of Rcd?}

The structure of Rcd is consistent with a role as a transacting anti-sense RNA. In a previous study the observation of $64 \%$ sequence complementarity between Rcd and the leucine attenuator led to the tentative suggestion that Rcd might exert its effect through modulation of intracellular leucine concentrations (Patient \& Summers, 1993). This now seems implausible because strain DS941 used in this study is incapable of leucine biosynthesis (it carries the leuB6 mutation) and 
(a)

cer

$35.4 \mathrm{~min}$

cer

$35.4 \mathrm{~min}$

cer

$35.4 \mathrm{~min}$

TTTACCTGTCTGCTGCCGTGATCGCGCTGAACGCGTTTT

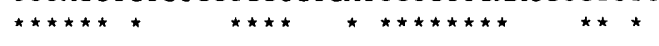

TTTACCGGGGATATGCCAGAAACGCGCTGAGTCAgTCTG

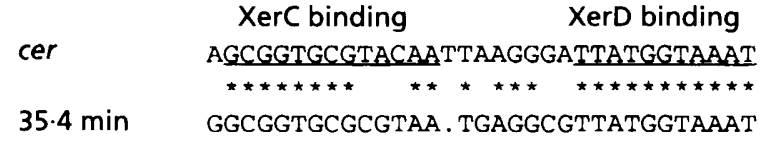

(b)

red

hns 3 -TAACATGCGTGGCGATTTTGCGCAAGTCGCGCT

Conserved region

rcd AGTGCCGTCGTCTGTCCATTTTTACCGTTGTTTGGTGGGCTTTTTGACGG
hns ctgaatttaaggetctattattacccCAACAAACCACCCCAATATAAGTT
-10

rcd CGCTAGCGCGGA-5

l 1

hns TGAGATTACTACAATGAGCGAAGCACTTAAAATTCTGAACAACATCCGTACT...
Fig. 8. Matches to the Rcd conserved sequence in the $E$. coli genome. (a) Sequence identity $\left(^{*}\right)$ between cer and the 35.4 min region. (b) Complementarity (*, Watson-Crick base pairing; |, T-G pairs) between DNA sequences encoding Red and the $5^{\prime}$ end of hns mRNA. The -35 and -10 sequences of the hns promoter are underlined, as is the ribosome-binding site (RBS). Untranscribed sequence upstream of the hns coding region is shown in lower case. Single letter amino acid codes indicate the $\mathrm{N}$ terminus of $\mathrm{H}-\mathrm{NS}$. yet remains Rcd-sensitive. The search for alternative potential targets has been hampered by the absence of knowledge of the extent of complementarity between Rcd and its target, or which part of the transcript is involved in the interaction. However, the discovery that a $15 \mathrm{nt}$ sequence within $\mathrm{Rcd}$ is conserved among its putative analogues suggested that this might be the active site of the transcript. Further support for this hypothesis came from the location of the sequence in the loop and upper stem of Rcd, which is the region of antisense RNAs which most often makes the primary interaction with the target.

Database searching identified three loci in the E. coli chromosome which matched at least 13 of the 15 conserved bases. Among these, hns mRNA looked the most plausible target. This was particularly interesting because $h n s$ mutants have been shown to have increased sensitivity to Rcd (Summers \& Rowe, 1997; Rowe \& Summers, 1999). However, the hypothesis that hns mRNA might be a target of Rcd was ruled out by experiments which showed the transcript had no effect on $h n s-l a c Z$ transcriptional and translational fusions. Neither of the two remaining chromosomal loci provided a credible mRNA target for Rcd so its mechanism of action remains ill-defined. Our failure to find a functional target could be due to differences between the AB1157 derivatives used in this work and the E. coli genome project strain MG1655 (Blattner et al., 1997). However, MG1655 is Rcd-sensitive (our unpublished data) so it seems unlikely that the strain lacks the Rcd target. If the $15 \mathrm{nt}$ conserved region is indeed the target interaction domain of Rcd our results imply that Rcd is not an antisense RNA. An alternative mechanism of action is a direct interaction between Rcd and a protein involved in cell division or its regulation.

\section{A chromosomal analogue of Red}

The discovery of a good match to the Rcd conserved sequence within a cer-like site near the replication terminus $(35.4 \mathrm{~min})$ raises the intriguing possibility that 
the chromosome encodes an Rcd analogue with a role in cell cycle control. There is poor conservation of the $\mathrm{P}_{\text {cer }}$ promoter between cer and the $35.4 \mathrm{~min}$ site but one would not expect plasmid and chromosomal versions of Rcd to be under identical transcriptional control. Certainly the region of the $35.4 \mathrm{~min}$ site corresponding to Rcd can be folded into an Rcd-like structure (data not shown) but we do not yet have any evidence whether a transcript is expressed or whether, if expressed, it would inhibit growth or cell division. Indeed, we cannot exclude the possibility that the $35.4 \mathrm{~min}$ site is the result of a chance plasmid integration event and is of no functional significance. However, if the chromosome does indeed encode an Rcd-like transcript with a role in cell cycle regulation it would explain the ease with which ColE1 seems able to control the cell cycle of its host. In retrospect, our failure to detect this locus when we used a conserved region probe against the gene mapping membrane is surprising. However, it is possible that the Kohara library (used to produce the membrane) is incomplete or that the chromosomal cer-like site is not universal and is absent from the Kohara source strain.

\section{ACKNOWLEDGEMENTS}

This work was supported by research studentships to M.E.S. and C.M. from the Medical Research Council and to H.L.W. from BBSRC. H.C. was supported by an MRC project grant to D. K.S. We thank Ian Viney, Charlie Hodgman and Marion Martin for their assistance with various aspects of the work.

\section{REFERENCES}

Andersen, J. \& Delihas, N. (1990). micF RNA binds to the 5 ' end of ompF mRNA and to a protein from Escherichia coli. Biochemistry 29, 9249-9256.

Bachmann, B. J. (1972). Pedigrees of some mutant strains of Escherichia coli $\mathrm{K}-12$. Bacteriol Rev' 36, 525-557.

Belasco, J. G., Nilsson, G., von Gabain, A. \& Cohen, S. N. (1986). The stability of $F$. coli gene transcripts is dependent on determinants localized to specific mRNA segments. Cell 46, 245-251.

Blakely, G., May, G., McCulloch, R., Arciszewska, L. K., Burke, M., Lovett, S. T. \& Sherratt, D. J. (1993). Two related recombinases are required for site-specific recombination at dif and cer in Escherichia coli K-12. Cell 75, 351-361.

Blattner, F. R., Plunkett, G., Bloch, C. A. \& 14 other authors (1997). The complete genome sequence of Escherichia coli K12. Science 277, 1453-1462.

Case, C. C., Simons, E. L. \& Simons, R. W. (1990). The IS10 transposase mRNA is destabilized during antisense RNA control. EMBO J 9, 12.59-1266.

Cohen, S. N. \& Hsu, L. (1972). Non chromosomal antibiotic resistance in bacteria : genetic transformation of Escherichia coli by R factor DNA. Proc Natl Acad Sci USA 69, 2110-2114.

Colloms, S. D., Sykora, P., Szatmari, G. \& Sherratt, D. J. (1990). Recombination at ColE1 cer requires the Escherichia coli xerC gene product, a member of the lambda integrase family of site specific recombinases. J Bacteriol 172, 6973-6980.

Hjalt, T. \& Wagner, E. G. H. (1992). The effect of loop size in antisense and target RNAs on the efficiency of antisense RNA control. Nucleic Acids Res 20, 6723-6732.

Kennedy, C. K. (1971). Induction of colicin production by high temperature or inhibition of protein synthesis. J Bacteriol 108, 10-19.

Kuempel, P. L., Henson, J. M., Dircks, L., Tecklenburg, M. \& Lim, D. F. (1991). dif, a recA-independent recombination site in the terminus region of the chromosome of Escherichia coli. New Biol 3, 799-811.

McKenny, K., Shimatake, H., Court, D., Schmeissner, U., Brady, C. \& Rosenberg, M. (1981). A system to study promoter and termination signals recognized by Escherichia coli RNA polymerase. In Gene Amplification and Analysis, pp. 383-415. Edited by J. G. Chirikjian \& T. S. Papa. Amsterdam: Elsevier.

Maniatis, T., Fritsch, E. F. \& Sambrook, J. (1982). Molecular Cloning: a Laboratory Manual. Cold Spring Harbor, NY : Cold Spring Harbor Laboratory.

Noda, A., Courtright, J. B., Denor, P. F., Webb, G., Kohara, Y. \& Ishihama, A. (1991). Rapid identification of specific genes in Escherichia coli by hybridization to membranes containing the ordered set of phage clones. Biotechniques 10, 474.

Patient, M. E. \& Summers, D. K. (1993). ColE1 multimer formation triggers inhibition of Escherichia coli cell division. Mol Microbiol 9, 1089-1095.

Rowe, C. D. \& Summers, D. K. (1999). The quiescent-cell expression system for protein synthesis in Escherichia coli. Appl Environ Microbiol 65, 2710-2715.

Stirling, C. J., Szatmari, G., Stewart, G., Smith, M. C. M. \& Sherratt, D. J. (1988). The arginine repressor is essential for plasmid-stabilizing site-specific recombination at the ColE1 cer locus. EMBO J 7, 4389-4395.

Stirling, C. J., Colloms, S. D., Collins, J. F., Szatmari, G. \& Sherratt, D. J. (1989). xerB, an Escherichia coli gene required for plasmid ColE1 site-specific recombination, is identical to $p e p A$, encoding aminopeptidase $A$, a protein with substantial similarity to bovine lens leucine aminopeptidase. EMBO J 8, 1623-1627.

Summers, D. K. (1998). Timing, self control and a sense of direction are the secrets of multicopy plasmid stability. Mol Microbiol 29, 1137-1145.

Summers, D. K. \& Rowe, D. C. (1997). Methods and means relating to quiescent cells and uses thereof. International Patent Application no. PCT/GB97/00731. Cambridge University Technical Services Ltd.

Summers, D. K. \& Sherratt, D. J. (1984). Multimerization of high copy number plasmids causes instability: ColE1 encodes a determinant essential for plasmid monomerization and stability. Cell 36, 1097-1103.

Summers, D. K. \& Sherratt, D. J. (1988). Resolution of ColE1 dimers requires a DNA sequence implicated in the threedimensional organisation of the cer site. EMBO J 7, 851-858.

Summers, D. K., Beton, C. W. H. \& Withers, H. L. (1993). Multicopy plasmid instability: the dimer catastrophe theory. Mol Microbiol 8, 1031-1038.

Tétart, F. \& Bouché, J. P. (1992). Regulation of the expression of the cell-cycle gene $f t s Z$ by DicF antisense RNA. Division does not require a fixed number of FtsZ molecules. Mol Microbiol 6, $61.5-620$.

Received 8 March 1999; revised 24 April 1999; accepted 5 May 1999. 